\title{
CONTROL DEL ENTRENAMIENTO DEPORTIVO EN DEPORTISTAS ESCOLARES
}

\section{CONTROL OF SPORTS TRAINING IN SCHOOL ATHLETES}

\begin{abstract}
Diego A. Alzate S. ${ }^{1}$, Carlos F. Ayala Z. ${ }^{2}$, Luis G. Melo B. ${ }^{3}$
${ }^{1}$ Profesor Universidad de Caldas, Lic. Educación Física, Esp. Mgr. Facultad de Ciencias para la Salud, Integrante Grupo de Investigación Cumanday. Avenida Paralela, calle 48 esquina, Manizales, Caldas, Colombia, e-mail: diego.alzate@ucaldas. edu.co; ${ }^{2}$ Profesor Universidad de Caldas, Lic. Educación Física, Especialista en Procesos Pedagógicos del Entrenamiento Deportivo, Magister en Educación, Ph.D. en Ciencias de la Motricidad, Integrante Grupo de Investigación Cumanday, e-mail: federico.ayala@ucaldas.edu.co; ${ }^{3}$ Tec. Educación Física, Ph.D Pedagogía - Entrenamiento deportivo, Facultad de Ciencias para la Salud, Integrante Grupo de Investigación Cumanday. Universidad de Caldas, Avenida Paralela, calle 48 esquina, Manizales, Caldas, Colombia, e-mail: luis.melo@ucaldas.edu.co
\end{abstract}

Rev. U.D.C.A Act. \& Div. Cient. 18(2): 321-328, Julio-Diciembre, 2015.

\section{RESUMEN}

Con el objetivo de determinar si a los deportistas escolares les fueron aplicados pruebas de control durante su proceso de entrenamiento para los IV Juegos Deportivos Escolares Centroamericanos y del Caribe, realizados en Armenia, Colombia, en 2013, fue desarrollado un estudio con enfoque cuantitativo, de corte transversal y alcance descriptivo, a una muestra por conveniencia de 233 deportistas escolares (102 hombres y 131 mujeres), quienes participaron en 12 deportes, representando a nueve países. El promedio de edad fue de 16,9 años $\pm 0,76$, con una edad deportiva de 7,26 años $\pm 3,27$. Fue administrada una encuesta elaborada por el grupo investigador y sometida a criterio de expertos. La información fue tratada con el programa SPSS (versión 17). Se encontró que a nueve de cada 10 deportistas les fueron aplicadas pruebas de control, siendo la más utilizada las físicas, con 169 respuestas, seguida de las médicas y obteniendo el menor número, las psicológicas. Hubo una fuerte asociación entre aplicación de las pruebas de control y deportistas, que pertenecen a ligas en sus respetivos países y medallería de oro obtenida, por diferentes países.

Palabras clave: Adolescente, atletas, pruebas de control, entrenamiento deportivo, formación deportiva.

\section{SUMMARY}

In order to determinate if school athletes are submitted to control tests during their training process for the IV Centro American and Caribbean Scholar Sport Games performed in Armenía, Colombia in 2013, a study with a quantitative approach, a cross-sectional and descriptive scope, was developed. A convenience sample of 233 school athletes, 102 men and 131 women, representing nine countries and participating in 12 sports, was performed. The average age was of $16.9 \pm 0.76$ years, with a sport activity time of $7.26 \pm 3.27$ years. A survey developed by a research group and submitted to expert judgement was administered. Participation was voluntary. Information was treated with SPSS program (version 17). It was found that 9 out of 10 athletes underwent control tests, being the most applied the physic ones with 169 answers, followed by medical tests and in a lowest number psychological ones. There was a strong association between the application of control tests and athletes who belong to leagues in their respective countries and the gold medals obtained during the games by different countries.

Key words: Adolescent, athletes, control test, sport training, school sport.

\section{INTRODUCCIÓN}

El control es considerado un componente muy importante dentro del proceso de entrenamiento y de competición del deportista, sirve como apoyo a su formación y permite verificar cómo va el proceso (Vasconcelos, 2000; Navarro \& Rivas, 2001; Borin et al. 2007a; Serrato, 2008; Calderón et al. 2011; Cuadrado et al. 2012). Valora, objetivamente, los aspectos que se relacionan con su estado, su nivel, la ejecución de la planificación y, especialmente, la eficacia en la competición, el alcance de los resultados establecidos y deseados (Reilly, 2001; Borin et al. 2007a; Cañadas \& Ibáñez, 2010). 
Además, permite modificarlo, si no se ajusta a los objetivos propuestos (Viru \& Viru, 2003; Thiess et al. 2004; Martin et al. 2004; Weineck, 2005; Borin et al. 2007b; Yanci \& Los Arcos, 2013), por lo que los procedimientos para el control del rendimiento deportivo son importantes y relevantes, para una calidad del proceso de entrenamiento deportivo (Alzate et al. 2012).

En este proceso de formación del deportista, el entrenador juega un papel importante, pues debe tener un gran conocimiento en relación al desarrollo deportivo del joven deportista (Freire, 2003; Gonçalves et al. 2012).

En consonancia con el papel del entrenador, Reilly (2001) y Murcia et al. (2004) expresan que los procesos de adaptación del deportista deben ser orientados al desarrollo, la valoración, el control y al análisis, por un grupo interdisciplinario de profesionales, que relacionen la información recolectada con: la capacidad funcional y los mecanismos de adaptación ante situaciones de solicitación máxima; la participación de las diferentes vías metabólicas de la producción de la energía necesaria para la actividad o el rendimiento físico-deportivo; la definición de su intensidad, la valoración de los mecanismos y la dinámica de la respuesta adaptativa, entre otros (Delgado, 2011; Campos, 2012).

Además de lo anterior, Pancorbo \& Blanco (1990) y Murcia et al. (2004) manifiestan que la realización del control del entrenamiento, a partir de los estadios de crecimiento, en relación con los diversos resultados de competición del deportista, se cuidará su salud, redundando en el mejoramiento del proceso, orientándolo para la consecución de los logros propuestos.

Con base en los argumentos expuestos, del por qué es importante la aplicación de pruebas de control durante los diferentes periodos de preparación del deportista escolar, se consideró necesario determinar si a los deportistas que participaron a los IV Juegos Deportivos Escolares Centroamericanos y del Caribe les fueron aplicados pruebas de control, además de establecer el tipo de pruebas aplicadas.

\section{MATERIALES Y MÉTODOS}

El tipo de estudio correspondió a un enfoque cuantitativo de corte transversal y de alcance descriptivo. Fueron encuestados por conveniencia 233 deportistas (102 hombres y 131 mujeres), debido a que no fue posible determinar los datos totales de los deportistas participantes en el evento.

La técnica empleada fue la encuesta, elaborada con base en una amplia referencia bibliográfica sobre la Teoría y Metodología del Entrenamiento Deportivo y bases de datos relacionados con trabajos de investigación científica, como Google
Académico, Redalyc, SciELO, Latindex, PubMed y Sport Discuss, además de páginas digitales sobre Educación Física y Deporte. La encuesta constaba de 26 preguntas, afines con seis tópicos de interés, que para efectos del presente artículo y en relación con el control de entrenamiento deportivo, se preguntó:

1) ¿A lo largo del proceso le han hecho pruebas de manera regular? Si - No;

2) ¿Cuáles son la pruebas más utilizadas?: A. Medidas corporales, B. Pruebas físicas, C. Habilidades deportivas, D. Valoraciones Médico deportiva, E. Valoración psicológica, que fueron cruzadas con alguna variables de interés, como: a) zona de residencia Rural - Urbana; b) tipo de institución educativa Pública - Privada; c) pertenece a liga deportiva: $\mathrm{Si}-\mathrm{No}$.

La encuesta fue elaborada por el grupo investigador, sometida a juicio de expertos nacionales y ajustada con base en una prueba piloto, realizada a deportistas no participantes en los juegos; ya que la muestra no fue probabilística ni representativa, no se pretende hacer inferencia de los resultados.

El tratamiento de los datos fue mediante la utilización del programa SPSS versión 17, utilizando frecuencia relativa y absoluta, promedio, desviación típica en algunas variables y chi-cuadrado para la significancia, cuando se cruzaron variables.

Los encuestados firmaron un consentimiento informado, en el cual, se les garantizaba la confidencialidad y el uso de la información, con fines académicos; además, la libertad para desistir de contestar alguna(s) preguntas o toda la encuesta, sin que eso tuviera consecuencia alguna. Se tuvo presente la Resolución 8430/1993 emitida por el Ministerio de Salud de la República de Colombia, Art. 11, dado que la solución del instrumento no implicaba riesgo para los participantes en el estudio.

\section{RESULTADOS Y DISCUSIÓN}

Características demográficas de la población: Se evaluaron 233 deportistas, 102 hombres $(43,8 \%)$ y 131 mujeres $(56,2 \%)$, cuyo promedio de edad era de $16,94 \pm 0,78$ y $16,81 \pm 0,75$ y edad deportiva de $7,32 \pm 332$ y $7,21 \pm 3,25$, para hombres y mujeres, respectivamente. Al agrupar por deportes, se registró que el $16,7 \%$ eran de resistencia; $30,9 \%$ de fuerza-velocidad; $24,9 \%$ de conjunto y $27,5 \%$ de combate.

Aplicación de pruebas de control por parte de los entrenadores durante el periodo de formación de los deportistas: Durante el proceso de formación del deportista y, específicamente, en la planificación del entrenamiento deportivo, 
se tienen en cuenta una gran variedad de elementos físicos, psicológicos, técnicos, tácticos e intelectuales, que se controlan y evalúan; por eso el control es considerado como el más importante (Ramos, 2001; Zhelyazkov, 2006), puesto que son evidencias de cómo va el proceso, determina los puntos fuertes y débiles de la preparación (Campos, 2012; Delgado, 1995; Jiménez et al. 2012) e identifica el verdadero potencial de rendimiento del deportista (Lanaro \& Silveira, 2001; Pearson et al. 2006).

En el proceso de formación y de planificación del entrenamiento del deportista es indispensable realizar diagnósticos iniciales, que indiquen cómo se encuentra el joven en sus primeras etapas, para la planeación adecuada de las cargas (Faigenbaum et al. 2001; Urdanpilleta et al. 2013), siendo importante y determinante el papel del entrenador en los controles y en el seguimiento, para el alcance de los objetivos propuestos (Morales, 2005; Pérez, 2008; Burgess \& Naughton, 2010; Pankhurst et al. 2013). Es así como al momento de preguntar al deportista escolar si en su proceso de formación les habían realizado algún tipo de pruebas de control (Figura 1), se halló que cerca de nueve de cada diez deportistas referenció que sí se le habían aplicado.

En esta investigación de Centro Americanos y del Caribe, no se presentan diferencias significativas $(p<0,05)$ en la aplicación de las pruebas, teniendo en cuenta el género, lo que indica que a ambos grupos les realizan controles, indistinta- mente de su sexo. Estas respuestas tienen consonancia con lo que se evidenció en los entrenadores, quienes asistieron a los deportistas en juegos Suramericanos Medellín - 2010, donde la mayoría empleaba pruebas de control. Estos resultados tienen estrecha relación con lo descrito por Alzate et al. (2012), donde al 9,7\%, no les hacían pruebas de control.

Como investigadores y con base en los autores anteriores (Alzate et al. 2012), comprendemos que, según lo encontrado, en el ámbito de América Latina, se presentan semejanzas en las propuestas de planificación deportiva, de características económicas, administrativas y formativas en torno a los procesos de formación deportiva y, específicamente, con el control del entrenamiento deportivo.

Se observa así, que se siguen presentando, en algunos entrenadores, dificultades para aplicar controles, pues se refleja en los resultados que no existe un seguimiento adecuado y continuo a los deportistas, para determinar cuál es su estado de preparación y de rendimiento, en relación a los propósitos planteados y que va en contra de llevar a cabo un proceso con objetividad, pues no hay previsión científica de los entrenadores, para conocer las características presentadas en el proceso y desde su inicio (Borin et al. 2007a), por posibles causas como: falta de conocimiento y reconocimiento de la importancia de los controles deportivos para los procesos y falencias y ausencias de infraestructuras, de materiales y de equipos adecuados, para la aplicación de los controles.

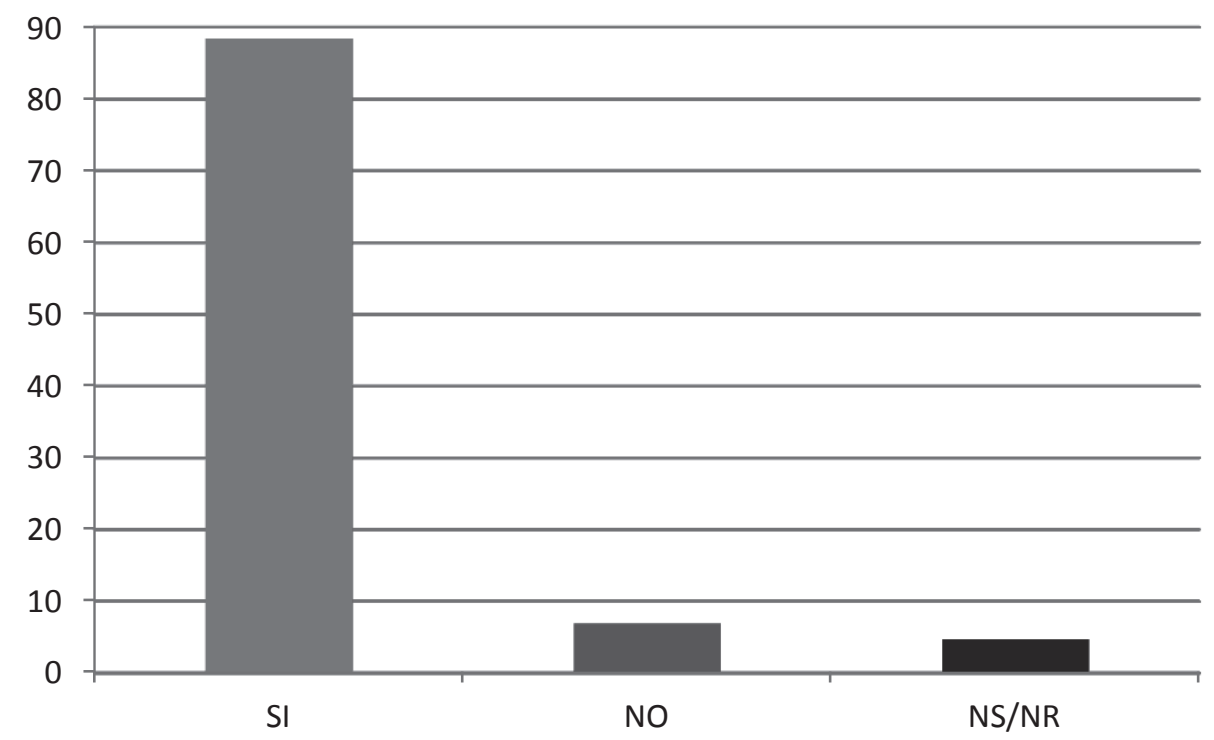

Figura 1. Porcentaje de deportistas, a quienes les fueron aplicadas pruebas de control, durante su proceso de formación. 
Aspectos de la preparacion del deportista escolar evaluados con las pruebas: Con relación a los aspectos de la preparación evaluados con las pruebas de control de los escolares deportistas (Figura 2), no a todos les aplicaron test de control, para determinar qué influencia estaba ejerciendo el plan de entrenamiento. Se debe tener en cuenta que, en las diferentes instituciones educativas, los deportistas escolares reciben la clase de educación física y solo algunos asisten o pueden asistir a escuelas de formación deportiva.

Es asi, como se muestra en la figura 2, que a solamente al $14 \%$ les fueron aplicados la totalidad de las pruebas de control, entre ellas, médicas, antropométricas, deportivas, fisicas y psicológicas, si se compara con los resultados presentados por Alzate et al. (2012), donde $11 \%$ de los entrenadores encuestados aplicaban la totalidad de éstas; se puede evidenciar, entonces, una leve diferencia, sin ser estadísticamente significativa, a favor de los escolares deportistas.

Al momento de cruzar las variables grupo de deporte con tipo de pruebas aplicadas, se hallaron diferencias altamente significativas $(p<0,01)$ en deportivas, donde el grupo de deporte que aplica con más frecuencia este tipo de pruebas son los de combate, con $58,7 \%$, seguidas de pelota, con $52,6 \%$ y, en último lugar, los de tiempo y marca, con $34,6 \%$; asimismo, se reportaron diferencias significativas $(p<0,05)$ en médicas, donde el grupo de deportes que aplica con más frecuencia este tipo de pruebas son los de combate, con $47,6 \%$, seguidas de tiempo y marca, con $32,7 \%$ y, con menor frecuencia, los de pelota, con 22,8\%; por último, se encontraron diferencias significativas en las antropométricas, donde los deportes de combate siguen aplicando -con mayor regularidad- este tipo de pruebas, con $42,9 \%$, seguido de tiempo y marca, con $37,5 \%$ y, finalmente, los de pelota, con $19,3 \%$.

Se encontró, además, que son realizadas, con mayor frecuencia, pruebas a los deportistas que pertenecen a ligas deportivas en sus respectivos países, que a aquellos que no dependen, evidenciándose diferencias altamente significativas $(p<0,01)$ en pruebas físicas $(p=0,004)$, seguidas de médicas y psicológicas $(p=0,006)$ y, en último lugar, con diferencias significativas, están las antropométricas $(p=0,033)$.

No se notó significancia entre el lugar de residencia (rural o urbana) en relación a la aplicación de pruebas de control, de forma general, ni con cada una de ellas.

Con los datos anteriores, se puede evidenciar una posible falta de conciencia de los entrenadores sobre la importancia de realizar pruebas de control de los procesos de planificación y del entrenamiento deportivo o tambien de la deficiencia en el apoyo y la asignación de recursos, para contratar, elaborar y aplicar los controles; además, en el proceso de selección y de clasificación a los eventos deportivos, en la diversidad de deportes y sus características o, incluso, de que algunos entrenadores y su equipo, no lo consideran necesario (Alzate et al. 2012), a pesar de ser deportistas y entrenadores de talla internacional.

Varios autores, como Platonov (2001), Gómez (2002), Zhelyazkov (2006) y Yanci \& Los Arcos (2013) rescatan la importancia del control del proceso de entrenamiento, como algo definitivo, para determinar el estado del deportista y del

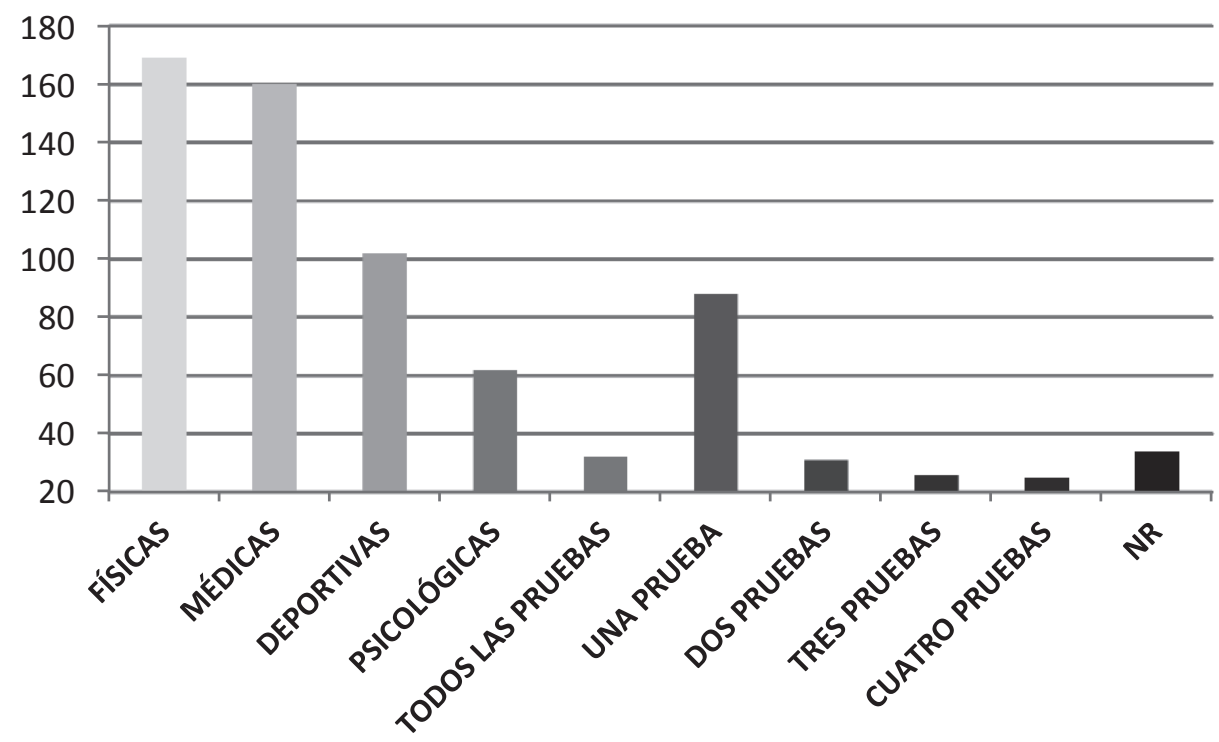

Figura 2. Aspectos de la preparacion del deportista escolar evaluados con las pruebas (número de respuestas). 
mismo proceso, mediante el análisis y la estructuración para garantizar el mejoramiento del entrenamiento y la consecución de altos logros, durante la competencia.

Según los encuestados, las pruebas de control que más se realizan son las físicas, seguidas de las médicas y, luego, las antropométricas (Figura 2), dándole así una amplia relevancia a los aspectos de la preparación fisica y fisiológica. En relación a lo anterior, se considera que existe una valoración del aspecto de la salud del deportista, como una manera de descubrir posibles problemas y contraindicaciones para la práctica deportiva, como: el atraso motor; problemas sicomotores, como el desvio de columna vertebral; enfermedades relacionadas con el Sistema Nervioso Central, cardio-vasculares, organicas y las relacionadas con el sentido vestibular (sentidos), entre otras (Lanaro \& Silveira, 2001).

Después de las anteriores pruebas, en escala de aplicación, se encuentran las deportivas, como se puede evidenciar en la figura 2; las que menos se aplicadan son las psicológicas, lo que permite pensar en un desconocimiento o falta de interes o la no presencia del profesional respectivo para realizar los test, reflejando la poca importancia que tiene este aspecto por parte de los entrenadores, dentro de la preparación del deportista, situación que se considera delicada, pues este último interfiere positiva o negativamente en el proceso, ya que se pueden valorar y controlar otros aspectos, como los trazos de personalidad, los niveles de ansiedad, de motiva- ción y de estrés, la autoconfianza, entre otras (González, 1997; Santos \& Pereira, 1997; Nicolai et al. 2004; Machado, 2011).

Asimismo, cuando se hace la comparación entre el número de pruebas aplicadas, se reflejó que el $37 \%$ solo utiliza una prueba de todas las referenciadas, demostrando, de esta manera, que no se presenta una planificación y estruturación de los controles dentro del proceso del entrenamiento deportivo, lo cual, contribuiría en el alcance de altos logros.

Medallero obtenido por parte de los deportistas, a quienes se le aplicaron las pruebas de control: Para hacer el análisis de las medallas obtenidas por países, en relación con la aplicación de las pruebas, se conformaron tres grupos de Naciones, teniendo en cuenta que hubo una diferencia en el tamaño de las delegaciones, a las cuales, se les aplicó el instrumento: los primeros tres: Colombia, Venezuela y Puerto Rico; los tres siguientes: México, Guatemala y Panamá y, por último, República Dominicana, Costa Rica y Nicaragua.

Comparando esta información con la aplicación de las pruebas de control durante el proceso de entrenamiento, se pudo evidenciar que el primer grupo, con un total de $57,5 \%$ de las pruebas realizadas, logró el $58,4 \%$ de las medallas de oro, otorgadas en las justas; seguido del grupo 2, con el $24 \%$ de las pruebas aplicadas, logró el 8,2, del medallero dorado y, por último, el grupo 3, con el 6,9\% de pruebas aplicadas, obtuvo el 6,0\%, de las medallas de oro (Figura 3).

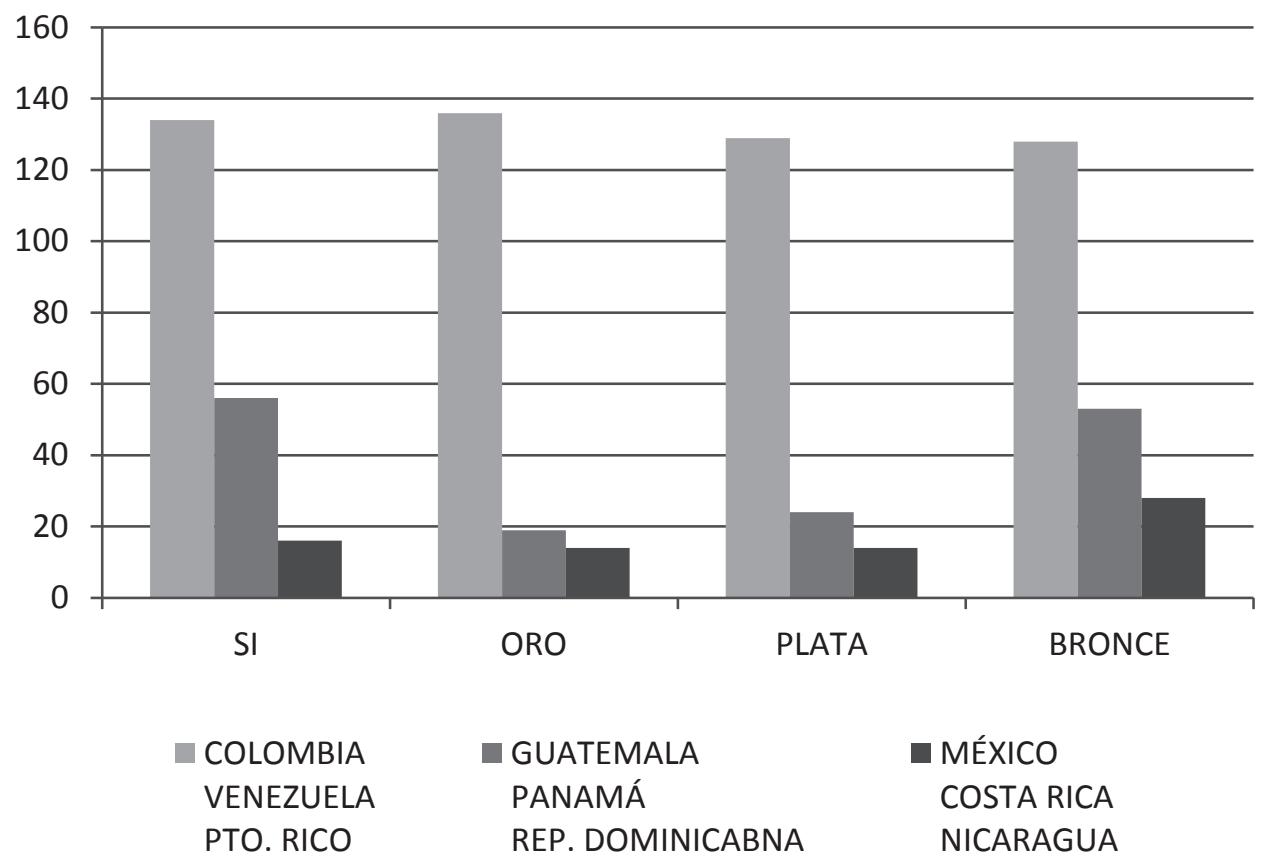

Figura 3. Medallas obtenidas por parte de los deportistas escolares, a quienes se les aplicó las pruebas de control (número de respuestas). 
Según los datos del medallero, se evidencia la utilidad y la relación entre la aplicación de las pruebas de control y los resultados obtenidos por los deportistas, permitiendo reorientar el proceso en busca de los objetivos propuestos, teniendo en cuenta la maduración del deportista escolar, a quien se le deben realizar test o pruebas, que correspondan a sus características. Cabe anotar, que además de la utilidad de las pruebas de control, hace parte de un conjunto de actividades no menos importantes, como lo son la nutrición, el entrenamiento, el perfil del entrenador, los recursos disponibles del deportista, la motivación y otros, que pueden influir en el proceso.

Se presentó una similitud con los resultados mostrados por Alzate et al. (2012), donde el 90,3\% de los entrenadores que respondieron positivamente a la pregunta, sobre la aplicación de pruebas de control, obtuvieron $88,3 \%$ de las medallas de oro; lo anterior, se relaciona con las medallas obtenidas, pues se evidenció que el grupo de entrenadores que aplican una o más pruebas tuvieron un mayor porcentaje de medallas de oro, que aquellas que solo aplicaron una sola prueba.

Agradecimientos: A los estudiantes de licenciatura en educación física y deportes de la Universidad del Quindío, Armenia. Financiación: La investigación fue financiada por la Vicerrectoría de Investigaciones y Postgrados de la Universidad de Caldas, Manizales, Colombia. Conflictos de interés: El manuscrito fue preparado y revisado con la participación de todos los autores, quienes declaramos que no existe conflicto de intereses que ponga en riesgo la validez de los resultados presentados.

\section{BIBLIOGRAFÍA}

1. ALZATE S, D.A.; AYALA Z., C.F.; MELO B., L.G. 2012. Control del entrenamiento deportivo en Juegos Suramericanos Medellín 2010. Rev. U.D.C.A Act. \& Div. Cient. (Colombia). 15(Supl.):87-95.

2. BORIN, J.; GOMES, A.; DOS SANTOS, G. 2007a. Preparação desportiva: aspectos do controle da carga de treinamento nos jogos coletivos. Rev. da Educação Física/UEM. (Brasil). 18 (1): 97-105. Disponible desde internet en: http://eduem.uem.br/ojs/ index.php/RevEducFis/article/view/3321/2391 (con acceso 26/6/2015).

3. BORIN, J.P.; PRESTES, J.; ALFANO M., N. 2007b. Caracterização, Controle e Avaliação: Limitações e Possibilidades no Âmbito do Treinamento Desportivo. Rev. Treinam. Desport. (Portugal). 8(1):6-11.
4. BURGESS, D.J.; NAUGHTON, G.A. 2010. Talent development in adolescent team sports: a review. Int. J. Sports Physiol. Perform. (US). 5(1):103-116.

5. CALDERÓN, J.; BENITO, P.; MELÉNDEZ, A.; GONZÁLEZ, M. 2011. Control biológico del entrenamiento de resistencia. Rev. Internacional de Ciencias del Deporte. (España). 2(2):65-87. Disponible desde Internet en: http://www.cafyd.com/REVISTA/art5n2a06. pdf (con acceso 3/03/2014).

6. CAMPOS V., M. 2012. Control den entrenamiento en fútbol: posibilidades de actuación. Rev. Entrenam. Deportivo RED. (España). Disponible desde internet en: http://revistaentrenamientodeportivo. com/?tag = control-entrenamiento (con acceso 10/03/2015).

7. CAÑADAS, M.; IBÁÑEZ, S.J. 2010. La planificación de los contenidos de entrenamiento de baloncesto en equipos de iniciación. Rev. Ciencias Deporte Ebalonmano.com (España). 6(1):49-65. Disponible desde Internet en: http://www.e-balonmano.com/ojs/ index.php/revista/index (con acceso 15/04/2014).

8. CUADRADO-REYES, J.; CHIROSA, R.L.; CHIROSA, R.I.; MARTIN-TAMAYO, I.; AGUILAR-MARTÍNEZ, D. 2012. La percepción subjetiva del esfuerzo para el control de la carga de entrenamiento en una temporada en un equipo de balonmano. Rev. Psicología del Deporte. (España). 21(2):331-339. Disponible desde Internet en: http://ddd.uab.cat/record/105919 (con acceso 25/6/2015).

9. DELGADO, M. 1995. Fundamentación anatómico funcional del rendimiento y del entrenamiento de la resistencia del niño y del adolescente. Rev. Motricidad: Eur. J. Human Movement (España). 1:97-110. Disponible desde internet en: https://doaj.org/article/2 3b23b0984f54346b04e02e05010bfb2 (con acceso 24/06/2015).

10. DELGADO, M.A. 2011. Evaluación funcional. Rev. Psicopatología Clínica, Legal y Forense. (España). 11:143-166. Disponible desde Internet en: http:// efisica-517-arcodelgado.blogspot.com/2011/10/ valoracion-funcional.html (con acceso 12/03/2014).

11. FAIGENBAUM, A.D.; LOUD, R.L.; O'CONNELL, J.; GLOVER, S.; O'CONNELL, J.; WESTCOTT, W.L. 2001. The Effects of Different Resistance Training Protocols on Muscular Strength and Endurance Development in Children. J. Strength Cond. Res. (US). 5(4):459-465. 
12. FREIRE, J.B. 2003. Pedagogia do Futebol. Autores Associados (Brasil). 98p.

13. GÓMEZ, C.P. 2002. Control del proceso de entrenamiento deportivo de alta competencia. Rev. Digital EFdeportes. (Argentina). Disponible desde Internet en: http://www.efdeportes.com/efd55/entren.htm. (con acceso 7/03/2014).

14. GONÇALVES, C.E.B.; RAMA, L.M.L.; FIGUEIREDO, A.B. 2012. Talent identification andspecialization in sport: an overview of some unanswered questions. Int. J. Sports Physiol. Perform. (US). 7(4):390-393.

15. GONZÁLEZ, J.L. 1997. Psicología del deporte. Biblioteca Nueva (España). 261p.

16. JIMÉNEZ-REYES, P.; CUADRADO-PEÑAFIEL, V.; GONZÁLEZ-BADILLO, J.J. 2012. Aplicación del CMJ para el control del entrenamiento en las sesiones de velocidad. Rev. Cultura, ciencia y deporte (España). Disponible desde Internet en: http://www.erevistas. csic.es/ficha_articulo.php?url=oai:ojs.ccd.ucam. edu:article/37\&oai_iden=oai_revista936 (con acceso: $24 / 06 / 2015)$.

17. LANARO, F.P.; SILVEIRA B., M.T. 2001. Detecção, seleção e promoção de talentos esportivos em ginástica rítmica desportiva: um estudo de revisão. Rev. Paulista Educ. Física (Brasil). 15(2):154-168.

18. MACHADO, A.P. 2011. Bases metodológicas da preparação física. Ícone Editora (Brasil). 248p.

19. MARTIN, D.; NICOLAUS, J.; OSTROWSKI, CH.; ROST, K. 2004 Metodología general del entrenamiento infantil y Juvenil. Paidotribo (España). 512p.

20. MORALES, A.A. 2005. La preparación del deportista: control y evaluación de la unidad de entrenamiento. Rev. Digital EFdeportes (Argentina). Disponible desde Internet en: http://www.efdeportes.com/efd86/ control.htm (con acceso 12/03/2014).

21. MURCIA, P.N.; TABORDA CH., J.; ANGEL, Z.L. 2004. Escuelas de formación deportiva y entrenamiento deportivo infantil. Kínesis (Colombia). 210p.

22. NAVARRO, F.; RIVAS, A. 2001. Planificación y Control del Entrenamiento en Natación. Gymnos (España). 153p.

23. NICOLAI, R.A.H.; DE ROSE, J.D.; SILVEIRA, B.M.T. 2004. Stress e nível competitivo: considerações so- bre jovenes praticantes de futsal. Rev. Bras. Ciências e Movimento. (Brasil). 12(4):83-87.

24. PANCORBO, A.; BLANCO, J. 1990. Consideraciones sobre el entrenamiento deportivo en la niñez y la adolescencia. Arch. Medicina Deporte. (Cuba). 7(27):309-314.

25. PANKHURST, A.; COLLINS, D.; MACNAMARA, Á. 2013 Talent development: linking the stakeholders to the process. J. Sports Sci (Gran Bretaña). 31(4):370380.

26. PEARSON, D.T.; NAUGHTON, G.A.; TODORE, M. 2006. Predictability of physiological testing and the role of maturation in talent identification for adolescent team sports. J. Sci. Med. Sport (Australia). 9(4):277287.

27. PÉREZ G., E. 2008. Las pruebas o tests en el deporte. Rev. Digital EFdeportes (Argentina). Disponible desde internet en: http://www.efdeportes.com/efd126/ las-pruebas-o-tests-en-el-deporte.htm (con acceso 12/03/2014).

28. PLATONOV, V.N. 2001. Teoría General del Entrenamiento Deportivo Olímpico. Paidotribo (España). 686p.

29. RAMOS B., S. 2001. Entrenamiento de la Condición Física. Kinesis (Colombia). 180p.

30. REILLY, T. 2001. Assessment of sports performance with particular reference to field games.Eur. J. Sport Science (Gran Bretaña). 1(3):1-12

31. SANTOS, S.G. dos; PEREIRA, S.A. 1997. Perfil do nível de ansiedade-traço pré-competitiva de atletas de esportes coletivos e individuais do Estado do Paraná. Rev. Movimento (Brasil). 4(6):3-13.

32. SERRATO R., M. 2008. Medicina del deporte. Universidad del Rosario (Colombia). 484p.

33. THIESS, G.; TSCHIENE, P.; NICKEL, H. 2004, Teoría y metodología de la competición deportiva. Paidotribo (España). 232p.

34. URDANPILLETA, A.; MARTINEZ-SANZ, J.; LOPEZGRUESO, R. 2013. Valoración Bioquímica del entrenamiento: Herramienta para el dietista-nutricionista deportivo. Rev. Española Nutr. Humana Dietética (España). 17(2):73-83. 
35. VASCONCELOS R., A. 2000. Planificación y Organización del Entrenamiento Deportivo. Paidotribo (España). 198p.

36. VIRU, A.; VIRU, M. 2003. Análisis y Control del Rendimiento Deportivo. Paidotribo (España). 286p.

37. WEINECK, J. 2005. Entrenamiento Total. Paidotribo (España). 688p.

38. YANCI IRIGOYEN, J.; LOS ARCOS LARUMBE, A. 2013.

Evolución del rendimiento aeróbico y anaeróbico en futbolistas profesionales tras la pretemporada. Rev. Cult. Ciencia y Deporte (España). Disponible desde Internet en: http://www.redalyc.org/articulo. oa?id=163028753006 (con acceso: 24/06/2015).

39. ZHELYAZKOV, T. 2006. Teoría y metodología del entrenamiento deportivo Feriva (Colombia). 458p.

Recibido: Marzo 25 de 2014

Aceptado: Agosto 3 de 2015

\section{Cómo citar:}

Alzate S., D.A.; Ayala Z., C.F.; Melo B., L.G. 2015. Control del entrenamiento deportivo en deportistas escolares. Rev. U.D.C.A Act. \& Div. Cient. 18(2): 321-328. 